\title{
Assessment of cryopreserved sperm functions of Korean native brindled cattle (Chikso) from different region research centers of Korea
}

\author{
Lei $\mathrm{Ma}^{1, \#}$, Dae-Jin Jung, ${ }^{2, \#}$, Eun-Ju Jung ${ }^{3}$, Woo-Jin Lee ${ }^{1}, \mathrm{Ju}^{-M i} \mathrm{Hwang}^{1}$, Jeong-Won Bae ${ }^{1}$, \\ Dae-Hyun $\mathrm{Kim}^{2}$, Jun Koo $\mathrm{Yi}^{2}$, Sang Moo Lee ${ }^{1}$, Jae Jung $\mathrm{Ha}^{2, *}$ and Woo-Sung Kwon ${ }^{1,3, *}$ \\ ${ }^{1}$ Department of Animal Science and Biotechnology, Kyungpook National University, Sangju 37224, Korea \\ ${ }^{2}$ Gyeongbuk Livestock Research Institute, Yeongju 36052, Korea \\ ${ }^{3}$ Department of Animal Biotechnology, Kyungpook National University, Sangju 37224, Korea
}

Received April 29, 2021

Revised June 17, 2021

Accepted June 23, 2021

*Correspondence

Woo-Sung Kwon

E-mail:wskwon@knu.ac.kr

ORCID

https://orcid.org/0000-0002-0848-7189

Jae Jung $\mathrm{Ha}$

E-mail: hjjggo@korea.kr

ORCID

https://orcid.org/0000-0001-6785-6346

"Lei Ma and Dae-Jin Jung contributed equally to this work.

\begin{abstract}
Sperm cryopreservation is an important method of assisted reproductive techniques and storing genetic resources. It plays a vital role in genetic improvement, livestock industrial preservation of endangered species, and clinical practice. Consequently, the cryopreservation technique is well organized through various studies, especially on Korean native cattle (Hanwoo). However, the cryopreservation technique of Korean native brindled cattle, which is one of the native cattle species in Korea, is not well organized. Therefore, it is necessary to develop a Supplementary Table technique for the cryopreservation of Korean native brindled cattle. For this purpose, it is important to first evaluate the quality of the currently produced cryopreserved sperm of Korean native brindled cattle. In this study, we randomly selected 72 individual Korean native brindled cattle semen samples collected from 8 different region research centers and used them to evaluate sperm functions. We focused on the quality evaluation of cryopreserved Korean native brindled cattle semen following the measurement of motion kinematics, capacitation status, intracellular ATP level, sperm motility, and cell viability. Then, the values of each of the eight groups were derived from various sperm parameters of nine individual samples, including sperm motility, kinematics, cellular motility, and intracellular ATP levels, which were used to compare and evaluate sperm function. Overall, differences in various sperm parameters were observed between most of the research centers. Particularly, the deviations of motility and motion kinematics were high according to the sample. Therefore, we suggest that it is necessary to develop a standard method for the cryopreservation of Korean native brindled cattle semen. We also suggest the need for sperm quality evaluation of the cryopreserved semen of Korean native brindled cattle before using artificial insemination to attain a high fertility rate.
\end{abstract}

Keywords: cryopreservation, Korean native brindled cattle, sperm functions, spermatozoa

\section{INTRODUCTION}

Artificial insemination (AI) is the most commonly used biotechnology for the genetic enhancement of cryopreserved sperm (Philpott, 1993). Cryopreservation is a technique that uses liquid nitrogen as a cold source to pre- 
serve cells for long periods of time (Karlsson and Toner, 1996). Sperm cryopreservation has aided the advancement of reproductive technologies, such as $\mathrm{AI}$ and in vitro fertilization (IVF). It is also used in a variety of clinical and research settings, including assisted reproductive technology, biological storage, and genetic improvement (Zavos, 1992; Curry, 1995). Cryopreservation technology solves the challenge of long-term preservation of semen and renders semen utilization unrestricted by time, geography, and the life of breeding animals. However, the genetic influence of AI is limited by the quality of the sperm (Yoon et al., 2015). A rapid, accurate, and objective evaluation of the functional state of frozen-thawed sperm is beneficial in the process of frozen semen research to facilitate the conservation of genetic resources of good breeding bulls and the establishment of gene pools, allowing complete utilization of the excellent genetic resources (Sansone et al., 2000). In contrast, it can accelerate the progression of breed improvement, improve breeding efficiency, and then maximize its optimal production performance and improve the economic benefits of animal husbandry (Andrabi and Maxwell, 2007). Cryopreservation of sperm from agriculturally and genetically important species would protect them from extinction.

Research conducted till date on semen cryopreservation in Hanwoo has been more extensive, and commercial frozen semen production is also more common. The technique of cryopreservation is well developed, the recovery rate is also good, and several studies also have been performed. Cryopreserved Hanwoo semen samples are well controlled and managed by a specific organization. Due to the limitation of genetic resources, freezing sperm obtained from Korean native brindled cattle would be a better method to conserve genetic resources. However, there is only limited experimental information on semen evaluation from Korean native brindled cattle in previous studies. Several research centers from each province of Korea are producing Korean native brindled cattle cryopreserved semen, and the methods of cryopreservation are different between each research center. A comprehensive management of the Korean native brindled cattle cryopreserved semen has not yet been implemented. Currently, a specific cryopreservation method has not been established. Therefore, quality evaluation of cryopreserved semen of the Korean native brindled cattle is necessary.

In this study, we randomly selected 72 individual Korean native brindled cattle samples from 8 different region research centers of Korea and used them for evaluating sperm functions. We focused on the quality evaluation of the cryopreserved Korean native brindled cattle semen following the measurement of capacitation status, motion kinematics, intracellular ATP level, sperm motility, and cell viability. Then, the values of each of the eight groups were derived from various sperm parameters of nine individual samples, including sperm motility, kinematics, cellular motility, and intracellular ATP levels, which were used to compare and evaluate the sperm functions.

\section{MATERIALS AND METHODS}

\section{Sample collection}

Semen samples were obtained from 72 individual Korean native brindled cattle from 8 different region research centers, stored on liquid nitrogen, and transferred to the laboratory.

\section{Sample preparation}

Sperm preparation was performed using the routine Percoll washing method as described previously (Lee et al., 2009; Brugnon et al., 2013). Frozen sperm samples were thawed for $20 \mathrm{~s}$ in a water bath at $39^{\circ} \mathrm{C}$. To remove extender debris, seminal plasma, cryoprotectant and diluent added during sperm cryopreservation, the samples were centrifuged for 20 minutes at $400 \times \mathrm{g}$ with a discontinuous Percoll density gradient of $1 \mathrm{~mL} 90 \%$ to $1 \mathrm{~mL} 45 \%$ Percoll. The top layer of the suspension was discarded after centrifugation. The sperm pellet was washed by centrifugation at $400 \times \mathrm{g}$ with $1 \mathrm{~mL}$ Tyrode's albumin lactate pyruvate (TALP) medium for $5 \mathrm{~min}$. The supernatant was extracted, after which $500 \mathrm{~L}$ of TALP medium was added. The TALP medium contained $1.0 \mathrm{mM}$ sodium pyruvate, $100 \mathrm{mM} \mathrm{NaCl}, 0.4 \mathrm{mM} \mathrm{MgCl} \cdot 6 \mathrm{H}_{2} \mathrm{O}, 3.1 \mathrm{mM} \mathrm{KCl}, 0.3 \mathrm{mM}$ $\mathrm{Na}_{2} \mathrm{HPO}_{4} \cdot 12 \mathrm{H}_{2} \mathrm{O}, 2.0 \mathrm{mM} \mathrm{CaCl} \cdot 2 \mathrm{H}_{2} \mathrm{O}, 25 \mathrm{mM} \mathrm{NaHCO}$, $21.6 \mathrm{mM}$ sodium lactate, and $0.6 \%$ bovine serum albumin.

\section{Computer-assisted sperm analysis}

Sperm motility and motion kinematics were evaluated using a computer-assisted sperm analysis (CASA) program (FSA2016, Medical supply, Seoul, Korea) with a CMOS image sensor and a $2048 \times 1536$ (300 M pixels), 60-frame camera (Medical supply, Seoul, Korea), and an OLYMPUS BX43 phase-contrast microscope (Olympus, Tokyo, Ja- 
pan) with a $10 \times$ objective phase-contrast mode. A $10-\mu \mathrm{L}$ smear of the sample was evaluated in a preheated $\left(37^{\circ} \mathrm{C}\right)$ Makler counting chamber (Sefi-Medical Instruments, Haifa, Israel). The movement of at least 250 sperm cells was recorded for each sample from random fields (> 5). The obtained images were analyzed to determine sperm motility (\%), progressive sperm motility (\%), rapid sperm motility (\%), medium sperm motility (\%), slow sperm motility (\%), curvilinear velocity (VCL, $\mu \mathrm{m} / \mathrm{s}$ ), straight line velocity (VSL, $\mu \mathrm{m} / \mathrm{s}$ ), average path velocity (VAP, $\mu \mathrm{m} / \mathrm{s}$ ), beat cross frequency $(\mathrm{BCF}, \mathrm{Hz})$, and amplitude of lateral head displacement (ALH, $\mu \mathrm{m})$

\section{Hoechst 33258 (H33258)/chlortetracycline fluorescence}

The H33258/CTC dual-staining method (combined Hoechst 33258/chlortetracycline fluorescence (CTC) assessment) was used to evaluate capacitation status. Samples were centrifuged at $400 \times \mathrm{g}$ for $5 \mathrm{~min}$. After removing the majority of the supernatant, $135 \mu \mathrm{L}$ of DPBS and $15 \mu \mathrm{L}$ of $\mathrm{H} 33258$ solution (10 g H33258/mL DPBS) were added to the remaining sample. After incubation for $3 \mathrm{~min}$ at room temperature (RT), $250 \mu \mathrm{L}$ of $2 \%(\mathrm{w} / \mathrm{v}$ ) polyvinylpyrrolidone in DPBS was added to the mixture to remove excess dye by layering. The sample was then washed by centrifugation at $400 \times \mathrm{g}$ for $5 \mathrm{~min}$. After the complete extraction of the supernatant, the pellet was resuspended in $50 \mu \mathrm{L}$ of CTC solution $(750 \mathrm{mM} \mathrm{CTC}$ in 5 $\mu \mathrm{L}$ buffer: $20 \mathrm{mM}$ Tris, $5 \mathrm{mM}$ cysteine, $130 \mathrm{mM} \mathrm{NaCl}, \mathrm{pH}$ 7.4) and in $50 \mu \mathrm{L}$ DPBS. The samples were then smeared onto slides in $10-\mu \mathrm{L}$ increments. For each sample, at least 400 spermatozoa were counted on each slide. For H33258 and CTC assessment, an OLYMPUS BX43 with epifluorescence illumination and ultraviolet BP 340-380/ LP 425 and BP 450-490/LP 515 excitation/emission filters were used (Olympus, Tokyo, Japan). Finally, the following four capacitation patterns were observed: live acrosomereacted sperm (AR pattern, no fluorescence over the head, or green fluorescence only in the postacrosomal region), live capacitated sperm (B pattern, the acrosomal region showed bright green fluorescence and the region showed a dark post-acrosomal), sperm that is not incapacitated and is alive (F pattern, bright green fluorescence that is evenly spread across the sperm head), and dead sperm (D pattern, blue fluorescence distributed uniformly over the sperm head).

\section{Intracellular ATP level}

Intracellular ATP generation was quantified using an ATP assay kit (ab83355, Abcam, Cambridge, UK) as instructed by the manufacturer. Briefly, Samples $\left(1 \times 10^{6}\right.$ cells $)$ were centrifuged at $10,000 \times \mathrm{g}$ for $5 \mathrm{~min}$, and removed the majority of the supernatants. The sample was then washed by $1 \times$ PBS $\left(4^{\circ} \mathrm{C}\right)$. The washed samples was added to 96-well plates. Before the experiment, the ATP reaction mixture was primed and equilibrated to RT. The reaction mixture was then added in equal volumes to each well and incubated at RT for 30 min shielded from light. Finally, luminescence was measured using a microplate reader at OD $570 \mathrm{~nm}$ (Gemini Em; Molecular Devices Corporation, Sunnyvale, CA, USA) and analyzed using the SoftMax Pro 7 software (Molecular Devices Corporation, Sunnyvale, CA, USA).

\section{Sperm viability}

The Cell Assay Kit was used to evaluate cell viability (ab112118, Abcam, Cambridge, UK). This kit uses a patented water-soluble dye that adjusts its absorption spectra in response to cellular reduction. The variation in absorption ratio is proportional to the number of live cells. The assay fluid was thawed and preheated to $37^{\circ} \mathrm{C}$ before the tests. Then, $100 \mu \mathrm{L}$ of spermatozoa and $20 \mu \mathrm{L}$ of assay solution were added to a 96-well plate. The sample was then incubated for $2 \mathrm{~h}$ at $37^{\circ} \mathrm{C}$ in $5 \% \mathrm{CO}_{2}$ in a light-protected incubator. Cell viability behavior, as well as absorbance at OD 570 and 605 nm, was measured (Gemini Em; Molecular Devices Corporation, Sunnyvale, CA, USA). The ratio of OD $570 \mathrm{~nm}$ to OD $605 \mathrm{~nm}$ was used to evaluate cell viability (SoftMax Pro 7; Molecular Devices Corporation, Sunnyvale, CA, USA).

\section{Statistical analysis}

The samples were divided into eight categories according to their source. In each category, numerical values were derived from various sperm parameters (such as motility, motion kinematics, capacitation state, viability, and ATP level) in nine individual samples. Data were interpreted using SPSS (Version 25.0, IBM, Armonk, NY, USA). One-way ANOVA was used to compare the various parameters of eight groups. Data were expressed as mean \pm SEM. $p$ values of $<0.05$ were considered to be statistically significant. 


\section{RESULTS}

\section{Sperm motion parameters}

The CASA program was used to monitor spermatozoa motility and motion kinematics after Percoll washing. Results showed that the deviation of motility (\%) and motion kinematics was high according to the sample ( $p<0.05$; Supplementary Table 1 and Fig. 1). No differences were observed between samples collected from the eight different region research centers in terms of medium motility and slow motility. The other eight parameters (sperm motility, progressive motility, rapid motility, VCL, VSL, VAP, BCF, and ALH) of samples collected from the G research center showed significantly higher values than those of samples collected from other research centers. Sperm motility and progressive sperm motility of samples from the $\mathrm{F}$ research center were significantly lower than those of samples from other research centers. VCL, VSL, VAP, $\mathrm{BCF}$, and ALH of samples from B, E, and F research centers were significantly lower than those of samples from other research centers $(p<0.05$; Supplementary Table 2 and Fig. 2).

\section{Assessment of sperm capacitation status}

CTC/H33258 dual staining was used to determine sperm capacitation status. Results showed that the proportions of $\mathrm{AR}, \mathrm{B}$, and $\mathrm{F}$ patterns were $0.72 \pm 0.59 \%, 11.25 \pm$ $5.13 \%$, and $88.03 \pm 5.32 \%$, respectively ( $p<0.05$; Fig. 3). The deviation of capacitation status was high according to the sample. The proportion of AR-pattern spermatozoa was the lowest $(0.03 \pm 0.10 \%)$ in samples collected from the $\mathrm{F}$ research center, whereas it was the highest $(1.45 \pm$ $0.27 \%$ in samples collected from the $G$ research center $(p<0.05$; Fig. 4). The proportion of B-pattern sperma-
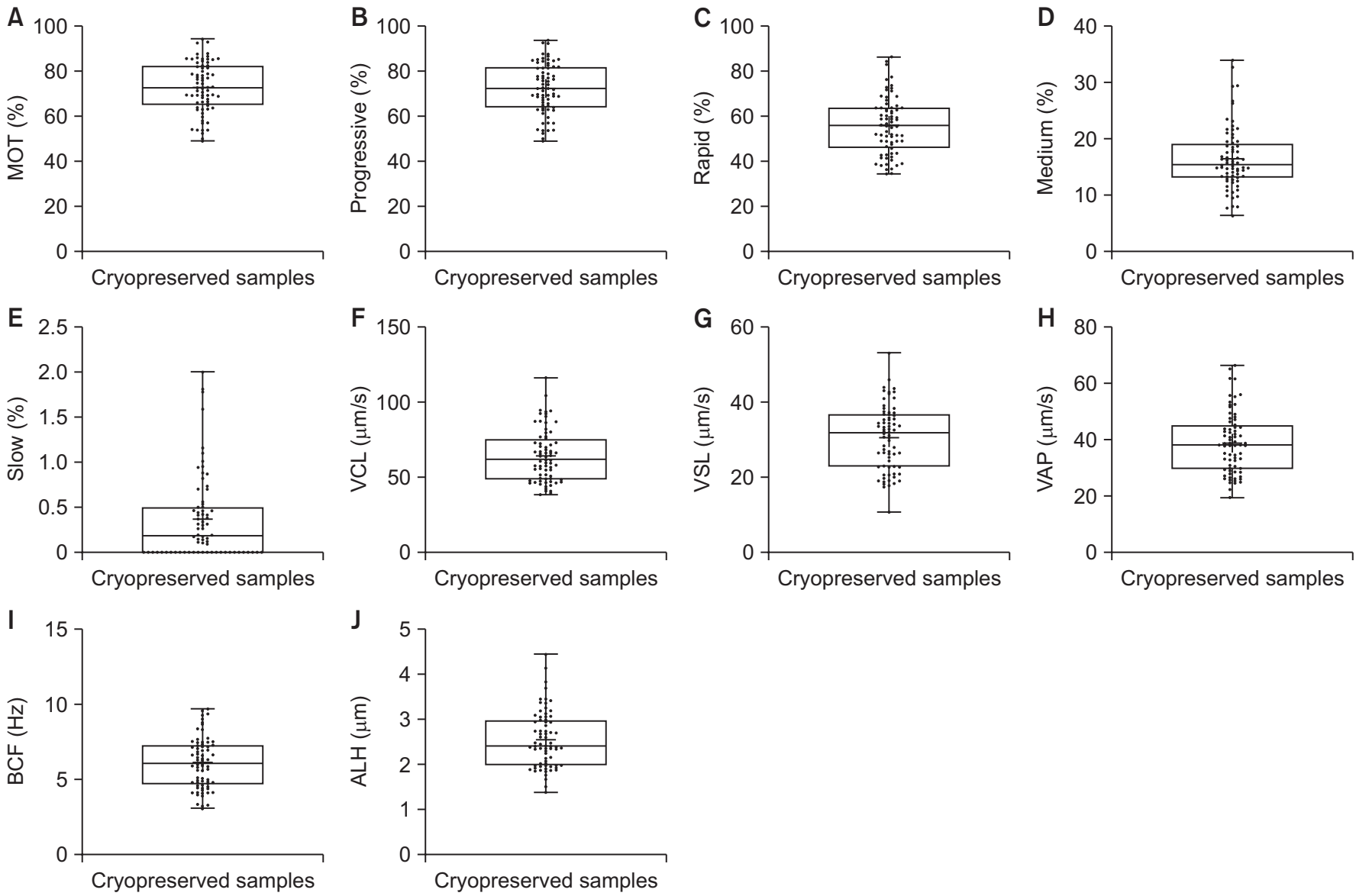

Fig. 1. Measurement of sperm motility of 72 individual Korean native brindled cattle after Percoll washing. (A) Sperm motility (\%). (B) Progressive sperm motility (\%). (C) Rapid sperm motility (\%). (D) Medium sperm motility (\%). (E) Slow sperm motility (\%). (F) Curvilinear velocity $(V C L, \mu \mathrm{m} / \mathrm{s})$. (G) Straight line velocity (VSL, $\mu \mathrm{m} / \mathrm{s})$. (H) Average path velocity (VAP, $\mu \mathrm{m} / \mathrm{s})$. (I) Beat cross frequency (BCF, Hz). (J) Amplitude of lateral head displacement $(\mathrm{ALH}, \mu \mathrm{m})$. 

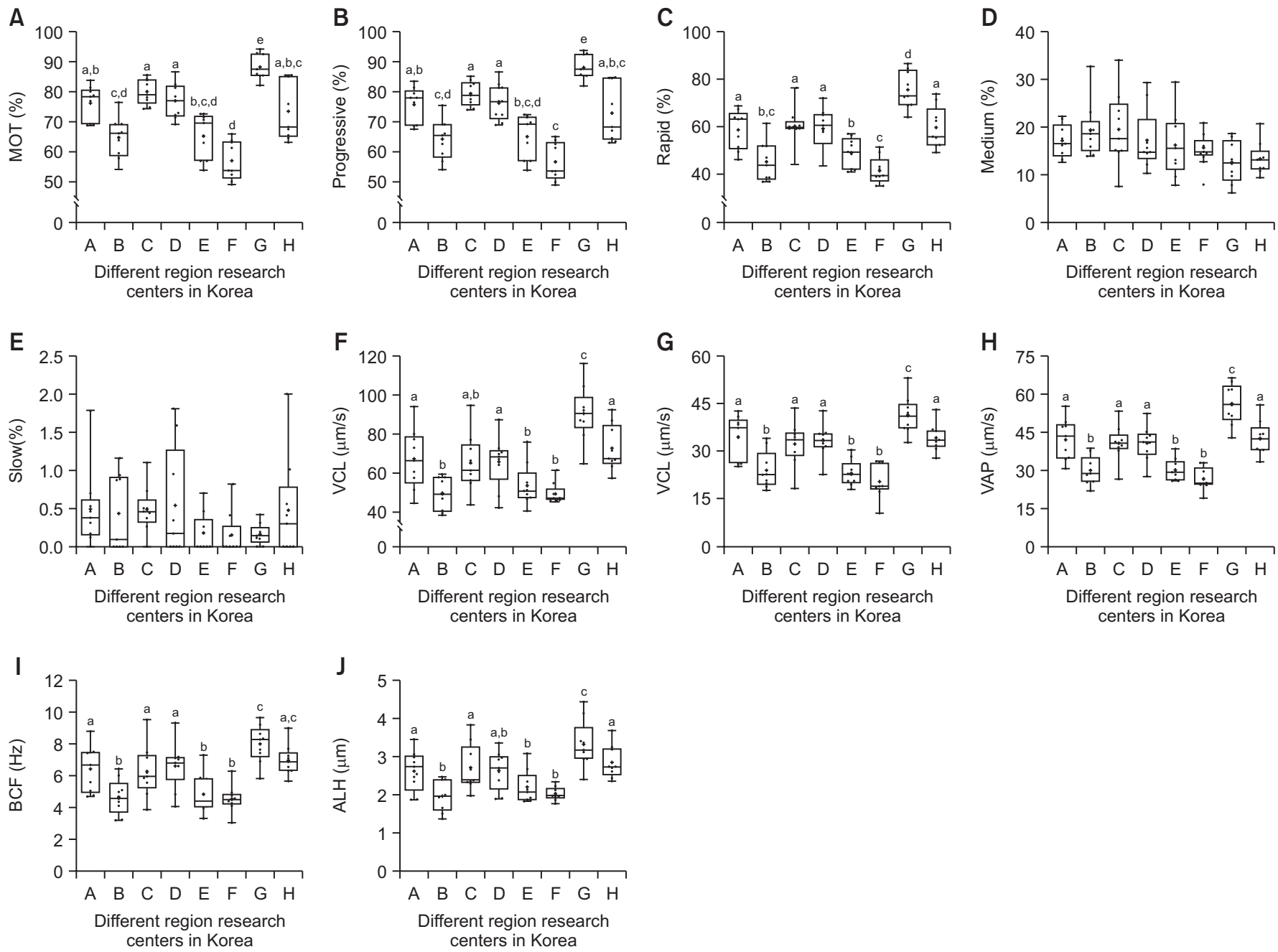

Fig. 2. Measurement of sperm motility of samples collected from eight different research centers. (A) Sperm motility (\%). (B) Progressive sperm motility (\%). (C) Rapid sperm motility (\%). (D) Medium sperm motility (\%). (E) Slow sperm motility (\%). (F) Curvilinear velocity (VCL, $\mu \mathrm{m} / \mathrm{s})$. (G) Straight line velocity (VSL, $\mu \mathrm{m} / \mathrm{s})$. (H) Average path velocity (VAP, $\mu \mathrm{m} / \mathrm{s}$ ). (I) Beat cross frequency (BCF, Hz). (J) Amplitude of lateral head displacement $(A L H, \mu m)$. Sperm motility and motion kinematics are presented as the mean \pm SE. Superscripts $a, b, c, d$ and e indicate significant differences between sperm parameters by one-way ANOVA of variance $(p<0.05, n=9)$.
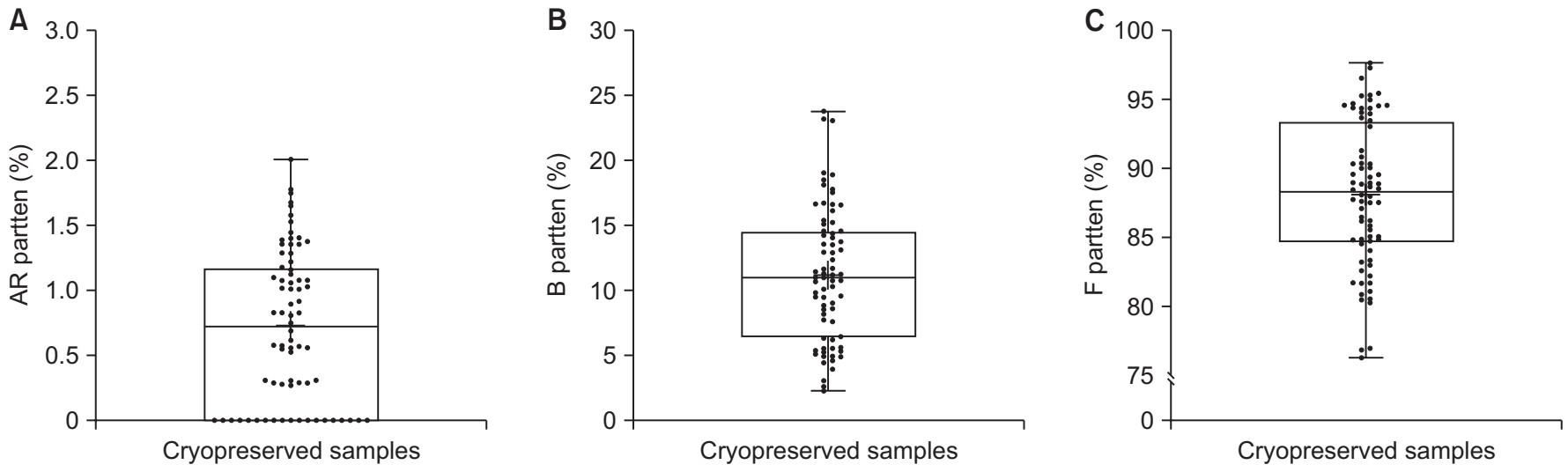

Fig. 3. Assessment of sperm capacitation status of 72 individual Korean native brindled cattle. (A) Patterns of live acrosome-reacted (AR pattern). (B) Patterns of live capacitated (B pattern). (C) Patterns of live noncapacitated (F pattern). 

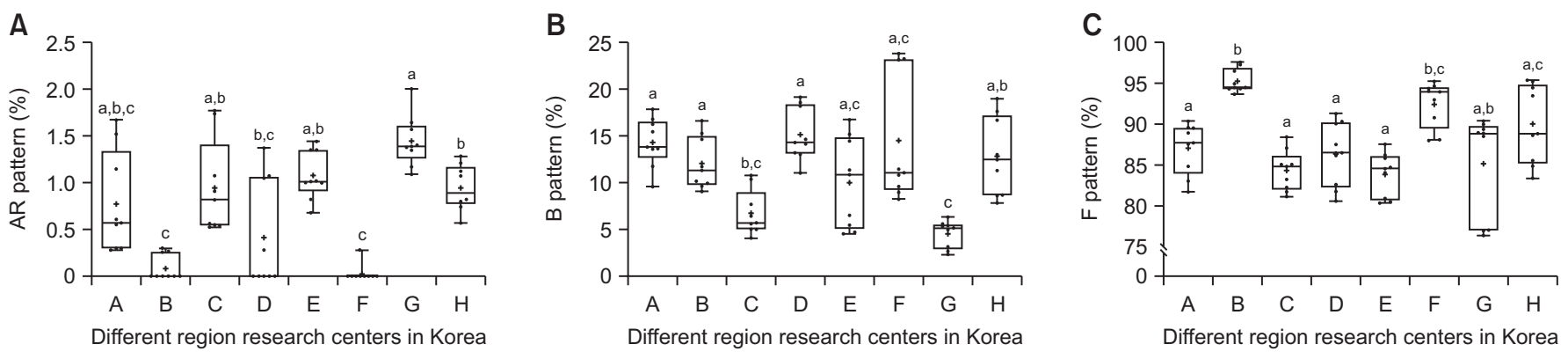

Fig. 4. Assessment of sperm capacitation status of samples collected from eight different research centers. (A) Patterns of live acrosomereacted (AR pattern). (B) Patterns of live capacitated (B pattern). (C) Patterns of live noncapacitated (F pattern). Data are presented as mean \pm SE. Superscripts $a, b$, and $c$ indicate significant differences between sperm parameters as assessed by one-way ANOVA ( $p<0.05$, $n=9)$.

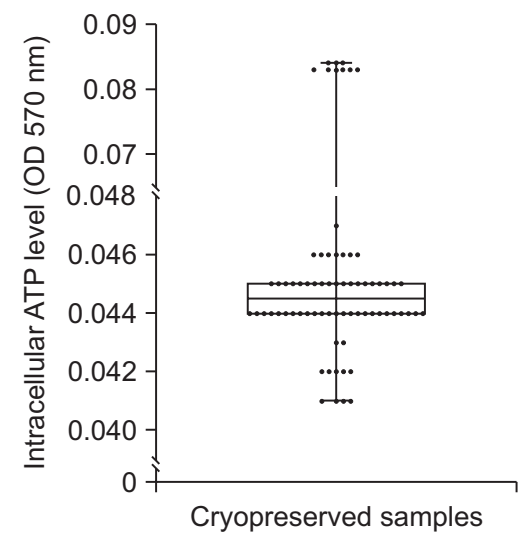

Fig. 5. ATP generation in spermatozoa of 72 individual Korean native brindled cattle.

tozoa was the lowest $(4.55 \pm 1.44 \%)$ in samples collected from the $G$ research center, whereas it was the highest $(15.13 \pm 2.82 \%)$ in samples collected from the D research center ( $p<0.05$; Fig. 4). Spermatozoa exhibiting the F pattern were detected in the highest proportion $(95.35 \pm$ $1.44 \%)$ at the B research center, whereas it was the lowest $(83.79 \pm 2.69 \%)$ at the E research center $(p<0.05$; Fig. 4).

\section{ATP generation in spermatozoa}

Quantitative ATP measurements were conducted using an ATP assay kit. The deviation of ATP measurements was high according to the sample ( $p<0.05$; Fig. 5 ). No differences were detected between samples collected from C, D, $\mathrm{E}$, and $\mathrm{H}$ research centers. The intracellular ATP levels of samples collected from $G(0.083 \pm 0.0005)$ and A (0.0460 $\pm 0.0005)$ research centers were significantly higher than those of samples collected from other research centers. The intracellular ATP levels of samples collected from $\mathrm{F}$ $(0.042 \pm 0.0005)$ and B $(0.044 \pm 0.0004)$ research centers

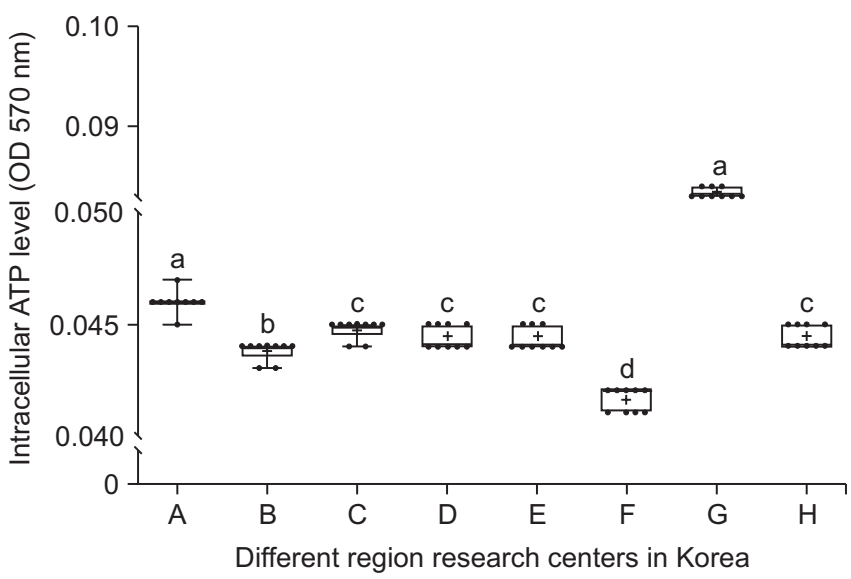

Fig. 6. ATP generation in spermatozoa of samples collected from eight different research centers. Data are presented as mean \pm SE. Superscripts $a, b, c$, and d indicate significant differences between sperm parameters as assessed by one-way ANOVA $(p<0.05$, $n=9)$.

were significantly lower than those of samples collected from other research centers ( $p<0.05$; Fig. 6).

\section{Cell viability}

Monitoring cell viability is one of the most important activities for comparing sperm functions. Results showed that the deviation of sperm cell viability was high according to the sample ( $p<0.05$; Fig. 7$)$. There were no differences between samples collected from C, D, and $\mathrm{H}$ research centers. However, significant differences were observed between samples collected from A, B, E, F, and $G$ research centers. The proportions of the cell viability of samples collected from G $(0.92 \pm 0.005)$ and A (0.89 \pm $0.004)$ research centers were significantly higher than those of samples collected from other research centers. The proportions of the cell viability of samples collected 


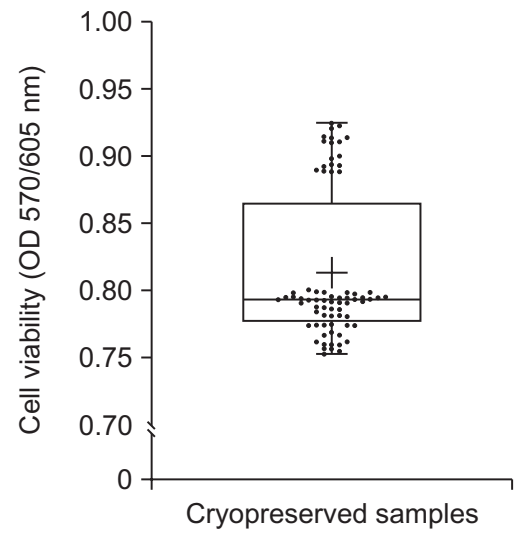

Fig. 7. Sperm cell viability of 72 individual Korean native brindled cattle.

from E $(0.78 \pm 0.003), \mathrm{B}(0.77 \pm 0.003)$, and F $(0.76 \pm$ $0.003)$ research centers were significantly lower than those of samples collected from other research centers $(p<0.05$; Fig. 8).

\section{DISCUSSION}

Currently, assessment of semen quality contributes substantially to the AI industry around the world (Larsson and Rodríguez-Martínez, 2000). In the bovine industry, > 70\% of cows are used for reproduction using frozen-thawed sperm obtained from superior genetic bulls through AI, but only $50 \%$ of full-term pregnancy complications are confirmed to be effective (Kastelic and Thundathil, 2008). As the quality of semen contributes significantly to subsequent fertility, it is essential to evaluate the quality of semen (Bonde, 1998). The research performed the more exact evaluation on bull semen quality certifies the breeding effects and the successful calvings. However, in previous research, only the limited data on Korean Native Brindled Cattle could be found. Therefore, we performed the frozen semen evaluation to provide an effective and widely applicable system for the assessment of Korean native brindled cattle semen as a foundation for improving semen evaluation standards.

Mammalian spermatozoa cannot fertilize oocytes before functional maturation. Maturity includes exercise, overactivation, capacitation, and AR (Suarez, 2008; Rahman et al., 2014). After ejaculation, spermatozoa in mammals gain motility. As only overactivated motile sperm can fertilize oocytes, sperm motility, and overactivation are considered as important factors for a successful

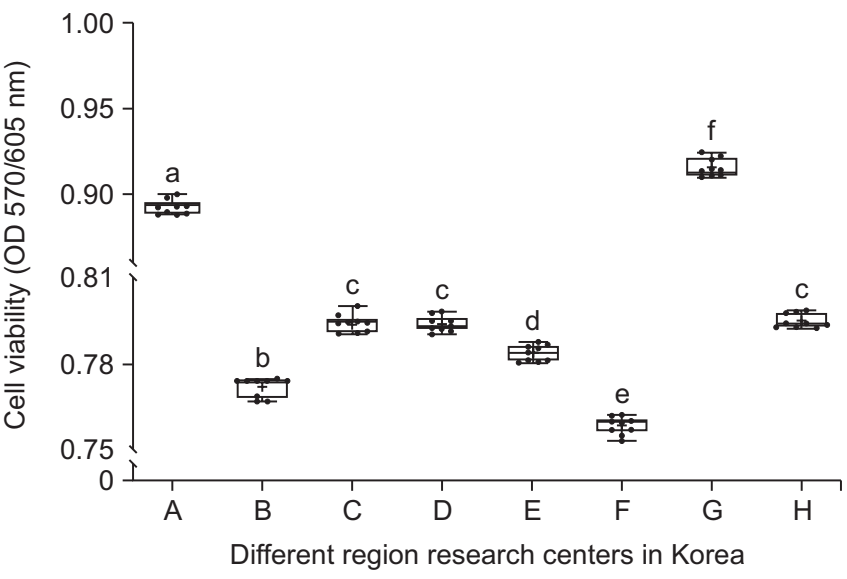

Fig. 8. Sperm cell viability of samples collected from eight different research centers. Data are presented as mean \pm SE. Superscripts $a, b, c, d$, and e indicate significant differences between sperm parameters as evaluated by one-way ANOVA $(p<0.05, \mathrm{n}=9)$.

pregnancy (Park, 2012; Rahman et al., 2013). The ratio of motile sperm is the important parameter on the semen evaluation. Therefore, it has been widely used in commercial and laboratory settings to evaluate sperm quality. Straightness, progressive trajectories, curvilinearity, and amplitude of lateral displacement were found in the sperm motility patterns, which are Supplementary Table for penetration into the cervical mucus of the female genital tract in vivo (David et al., 1981; Serres et al., 1984). The CASA system was used to evaluate motility and other kinematic parameters in this study; the analysis have been extensively used to determine the quality of sperm in animal species. The kinematic values determined for each spermatozoon include the sperm head's velocity of moves, the width of the sperm head's trajectory, and the frequency of the sperm head's directional movement (David et al., 1981). Our results showed that the sperm motility, progressive motility, rapid motility, VCL, VSL, VAP, BCF, and ALH of samples collected from the $G$ research center were significantly higher than those of samples collected from other research centers ( $p$ $<0.05$; Supplementary Table 2 and Fig. 2). Sperm motility and progressive sperm motility of samples collected from the $F$ research center were significantly lower than those of samples collected from other research centers. VCL, VSL, VAP, BCF, and ALH of samples collected from $\mathrm{B}, \mathrm{E}$, and $\mathrm{F}$ research centers were significantly lower than those of samples collected from other research centers. Under both in vitro and in vivo conditions, effective fertilization requires completion of spermatozoa capacita- 
tion at the appropriate time. In fact, fertilization is a complicated series of events involving several regulatory steps. According to a review of the literature, no specific and direct methods exist for determining the fertilization capacity of spermatozoa, and detecting the fertilization characteristics of most of the spermatozoa is quite difficult (Gillan et al., 2005). In this regard, determining the spermatozoa's capacitation status is believed to be an important indicator of the quality of the sperm. The status of spermatozoa capacitation can be determined directly by monitoring calcium regulation changes with chlortetracycline, a fluorescent antibiotic. The highly fluorescent $\mathrm{Ca}^{2+}$ complexes bind to the cell membrane, resulting in four different spermatozoa staining patterns (Zaneveld et al., 1991; Fraser and McDermott, 1992). The acrosome reaction occurs during fertilization, and the sperm loses the integrity of the head portion of the membrane that promotes sperm-zona binding after capacitation (Luconi et al., 1996; Kirichok et al., 2006). Our results showed that samples collected from the F research center had the lowest proportion of AR-pattern spermatozoa, whereas those collected from the $G$ research center had the highest proportion. The proportion of B-pattern spermatozoa was the lowest in samples collected from the E research center, whereas it was the highest in samples collected from the B research center. Regarding the proportion of F-pattern spermatozoa, it was the lowest at the B research center but the highest at the E research center $(p<0.05$; Fig. 4).

Mammalian sperm must gradually move in a certain period of time to fertilize the oocyte (Mortimer, 1990). As the ejaculated spermatozoa demand substantial amounts of energy to facilitate in vivo transport through the female reproductive tract and for both in vitro and in vivo penetration of the oocyte zona pellucida (Mortimer, 1990; Mukai and Okuno, 2004), it is important to note that sperm motility depends primarily on the availability of intracellular ATP. Depletion of ATP during capacitation is attributed to the loss of ATP required to maintain sperm motility and activate cAMP (Wishart and Palmer, 1986). It has been suggested that the energy required to regulate sperm motility is provided by ATP, and ATP is primarily produced by mitochondrial respiration. Therefore, if mitochondrial respiration cannot synthesize ATP, it may reduce sperm motility. Mitochondria play a central role in energy metabolism and regulate sperm motility, AR, and sperm oocyte fusion (Mukai and Okuno, 2004). Our results demonstrated that the intracellular ATP levels of samples collected from $G$ and A research centers were significantly higher than those of samples collected from other research centers ( $p<0.05$; Fig. 6).

Sperm viability is the key factor for determining semen quality; low sperm viability can directly affect male fertility (Tsujii et al., 2002; Kaur and Bansal 2004). In our study, the cell viability of samples collected from $G$ and A research centers was significantly higher than that of samples collected from other research centers. However, the cell viability of samples collected from E, B, and F research centers was significantly lower than that of samples collected from other research centers $(p<0.05$; Fig. 8).

The deviations of sperm motility (\%) and motion kinematics were high according to the semen sample. In addition, differences in various sperm parameters were observed between most of the research centers. It may be judged methods for cryopreservation of Korea native brindled cattle are different in each research center because a Supplementary Table standard protocol for cryopreservation of Korea native brindled cattle does not exist. Therefore, we suggest that it is necessary to develop a standard method for the cryopreservation of Korean native brindled cattle semen. We also suggest that it is important to evaluate the sperm quality of the cryopreserved semen of Korean native brindled cattle before performing AI to attain a high fertility rate. Furthermore, these findings may provide a useful model for the development of semen cryopreservation strategies in other endangered species.

\section{CONFLICTS OF INTEREST}

No potential conflict of interest relevant to this article was reported.

\section{ACKNOWLEDGEMENTS}

This study was conducted with the support of the 2020 Gyeongsangbuk-do agricultural and fishery R\&D activation project. 


\section{AUTHOR CONTRIBUTIONS}

Conceptualization: Lei Ma, Dae-Jin Jung, Jae Jung Ha, Woo-Sung Kwon

Data curation: Jae Jung Ha, Woo-Sung Kwon

Formal analysis: Lei Ma, Dae-Jin Jung,Eun-Ju Jung,

Woo-Jin Lee, Ju-Mi Hwang, Jeong-Won Bae, Dae-Hyun Kim, Jun Koo Yi, Sang Moo Lee, Jae Jung Ha, Woo-Sung Kwon

Funding acquisition: Jae Jung Ha, Woo-Sung Kwon

Investigation: Lei Ma, Dae-Jin Jung,Eun-Ju Jung,

Woo-Jin Lee, Ju-Mi Hwang, Jeong-Won Bae, Dae-Hyun Kim, Jun Koo Yi, Sang Moo Lee

Methodology: Lei Ma, Dae-Jin Jung, Jae Jung Ha,

Woo-Sung Kwon

Project administration

Resources: Gyeongbuk Provincial Government

Software: Jae Jung Ha, Woo-Sung Kwon

Supervision: Jae Jung Ha, Woo-Sung Kwon

Validation: Jae Jung Ha, Woo-Sung Kwon

Visualization: Jae Jung Ha, Woo-Sung Kwon

Writing - original draft: Lei Ma, Dae-Jin Jung, Jae Jung Ha,

Woo-Sung Kwon

Writing - review \& editing: Jae Jung Ha, Woo-Sung Kwon

\section{AUTHOR'S POSITION AND ORCID NO.}

L Ma, M.S. Student, https://orcid.org/0000-0002-3011-8080

DJ Jung, Researcher, https://orcid.org/0000-0002-2556-6241

EJ Jung, B.S. Student, https://orcid.org/0000-0001-7122-2004

WJ Lee, M.S. Student, https://orcid.org/0000-0002-4690-7148

JM Hwang, M.S. Student, https://orcid.org/0000-0003-1776-9759

JW Bae, Ph.D. Student, https://orcid.org/0000-0002-7030-7767

DH Kim, Researcher, https://orcid.org/0000-0002-4820-4438

JK Yi, Researcher, https://orcid.org/0000-0003-2593-6529

SM Lee, Professor, https://orcid.org/0000-0003-2510-2591

JJ Ha, Researcher, https://orcid.org/0000-0001-6785-6346
WS Kwon, Professor,

https://orcid.org/0000-0002-0848-7189

\section{SUPPLEMENTARY MATERIALS}

Supplementary materials can be found via https://doi.12750/ JARB.36.2.106.

\section{REFERENCES}

Andrabi SM and Maxwell WM. 2007. A review on reproductive biotechnologies for conservation of endangered mammalian species. Anim. Reprod. Sci. 99:223-243.

Bonde JP, Ernst E, Jensen TK, Hjollund NH, Kolstad H, Henriksen TB, Scheike T, Giwercman A, Olsen J, Skakkebaek NE. 1998. Relation between semen quality and fertility: a population-based study of 430 first-pregnancy planners. Lancet 352:1172-1177.

Brugnon F, Ouchchane L, Pons-Rejraji H, Artonne C, Farigoule M, Janny L. 2013. Density gradient centrifugation prior to cryopreservation and hypotaurine supplementation improve post-thaw quality of sperm from infertile men with oligoasthenoteratozoospermia. Hum. Reprod. 28:2045-2057.

Curry MR. 1995. Cryopreservation of semen from domestic livestock. Methods Mol. Biol. 38:189-197.

David G, Serres C, Jouannet P. 1981. Kinematics of human spermatozoa. Gamete Res. 4:83-95.

Fraser L and McDermott CA. 1992. Ca(2+)-related changes in the mouse sperm capacitation state: a possible role for $\mathrm{Ca}(2+)$-ATPase. J. Reprod. Fertil. 96:363-377.

Gillan L, Evans G, Maxwell WM. 2005. Flow cytometric evaluation of sperm parameters in relation to fertility potential. Theriogenology 63:445-457.

Karlsson JO and Toner M. 1996. Long-term storage of tissues by cryopreservation: critical issues. Biomaterials 17:243-256.

Kastelic JP and Thundathil JC. 2008. Breeding soundness evaluation and semen analysis for predicting bull fertility. Reprod. Domest. Anim. 43 Suppl 2:368-373.

Kaur P and Bansal MP. 2004. Influence of selenium induced oxidative stress on spermatogenesis and lactate dehydrogenase-X in mice testis. Asian J. Androl. 6:227-232.

Kirichok Y, Navarro B, Clapham DE. 2006. Whole-cell patchclamp measurements of spermatozoa reveal an alkalineactivated $\mathrm{Ca}^{2+}$ channel. Nature 439:737-740.

Larsson B and Rodríguez-Martínez H. 2000. Can we use in vitro fertilization tests to predict semen fertility? Anim. Reprod. Sci. 60-61:327-336.

Lee HL, Kim SH, Ji DB, Kim YJ. 2009. A comparative study of Sephadex, glass wool and Percoll separation techniques on sperm quality and IVF results for cryopreserved bovine semen. J. Vet. Sci. 10:249-255.

Luconi M, Krausz C, Forti G, Baldi E. 1996. Extracellular calcium negatively modulates tyrosine phosphorylation and 
tyrosine kinase activity during capacitation of human spermatozoa. Biol. Reprod. 55:207-216.

Mortimer D. 1990. Objective analysis of sperm motility and kinematics. In: Keel BA, Webster BW (Eds.), Handbook of the Laboratory Diagnosis and Treatment of Infertility, CRC Press, Boca Raton, pp. 97-133.

Mukai C and Okuno M. 2004. Glycolysis plays a major role for adenosine triphosphate supplementation in mouse sperm flagellar movement. Biol. Reprod. 71:540-547.

Park YJ, Kwon WS, Oh SA, Pang MG. 2012. Fertility-related proteomic profiling bull spermatozoa separated by percoll. J. Proteome Res. 11:4162-4168.

Philpott M. 1993. The dangers of disease transmission by artificial insemination and embryo transfer. Br. Vet. J. 149:339369.

Rahman MS, Kwon WS, Pang MG. 2014. Calcium influx and male fertility in the context of the sperm proteome: an update. Biomed Res. Int. 2014:841615.

Rahman MS, Lee JS, Kwon WS, Pang MG. 2013. Sperm proteomics: road to male fertility and contraception. Int. J. Endocrinol. 2013:360986.

Sansone G, Nastri MJ, Fabbrocini A. 2000. Storage of buffalo (Bubalus bubalis) semen. Anim. Reprod. Sci. 62:55-76.
Serres C, Feneux D, Jouannet P, David G. 1984. Influence of the flagellar wave development and propagation on the human sperm movement in seminal plasma. Gamete Res. 9:183-195.

Suarez SS. 2008. Control of hyperactivation in sperm. Hum. Reprod. Update 14:647-657.

Tsujii T, Kamai T, Moriguchi H, Hosoya Y, Honda M, Yamanishi T, Yoshida K, Kitahara S, Tachibana Y. 2002. [Seminal lactate dehydrogenase $\mathrm{C} 4$ ( $\mathrm{LDH}-\mathrm{C} 4)$ isozyme activity in infertile men]. Hinyokika Kiyo 48:193-197. Japanese.

Wishart GJ and Palmer FH. 1986. Correlation of the fertilising ability of semen from individual male fowls with sperm motility and ATP content. Br. Poult. Sci. 27:97-102.

Yoon SJ, Kwon WS, Rahman MS, Lee JS, Pang MG. 2015. A novel approach to identifying physical markers of cryo-damage in bull spermatozoa. PLoS One 10:e0126232.

Zaneveld LJ, De Jonge CJ, Anderson RA, Mack SR. 1991. Human sperm capacitation and the acrosome reaction. Hum. Reprod. 6:1265-1274.

Zavos PM. 1992. Preparation of human frozen-thawed seminal specimens using the SpermPrep filtration method: improvements over the conventional swim-up method. Fertil. Steril. 57:1326-1330. 